\title{
Fasten als Ergänzung zur Chemotherapie
}

\author{
Es häufen sich Hinweise darauf, dass Fasten gesunde Zellen vor zytotoxischen \\ Einflüssen schützt, Tumorzellen dagegen zusätzlich stresst. Ausgehend von \\ dieser vor allem im Tiermodell nachgewiesenen "differenziellen Stressresis- \\ tenz" wurde in einer Studie die Wirkung einer Fastenintervention in Kombina- \\ tion mit einer Chemotherapie untersucht.
}

nsgesamt 13 Frauen mit HER2-negativem Mammakarzinom Stadium II und III, für die sechs dreiwöchige Chemotherapiezyklen (Docetaxel, Doxorubicin und Cyclophosphamid) vorgesehen waren, wurden in die Interventionsgruppe (Fasten $24 \mathrm{~h}$ vor bis $24 \mathrm{~h}$ nach Chemotherapie) oder Kontrollgruppe (gesunde Ernährung im gleichen Zeitraum) randomisiert. Nebenwirkungen und Blutparameter wurden vor Randomisierung sowie vor und sieben Tage nach jeder Chemotherapie erfasst. DNA-Doppelstrangbrüche verschiedener peripher mononukleärer Blutzellen (PBMC) wurden mittels $\gamma$-H2AX-Quantifizierung bestimmt.

Die 48-stündige Fastenperiode, in der nur Wasser, Tee und Kaffee ohne Zucker erlaubt waren, wurde gut toleriert. Zwei von sieben Patientinnen beendeten die Intervention vorzeitig nach dem dritten Zyklus aufgrund von Pyrosis oder rezidivierender fiebriger Neutropenie. Diese Beschwerden blieben jedoch auch während der restlichen Zyklen ohne Fasten bestehen. Hinsichtlich Zahl und Schwere der Nebenwirkungen unterschieden sich die Gruppen nicht. Unterschiede ergaben sich beim Insulinspiegel, der nur in der Kontrollgruppe signifikant gegenüber dem Ausgangswert anstieg, und beim IGF-1-Spiegel (,insulin-like

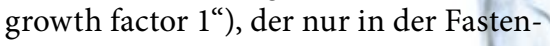
gruppe abfiel. Bei den hämatologischen Parametern zeigten sich zu Beginn jeder Chemotherapie keine Unterschiede. Sie ben Tage nach Chemotherapie waren die Erythrozyten- und Thrombozytenzahlen in der Fastengruppe jedoch höher als in der Kontrollgruppe. Für Leukozyten und Neutrophile ergaben sich keine Unterschiede. Eine Zunahme an Doppelstrangbrüchen war nur in der Kontrollgruppe nachweisbar. Dort war in manchen PBMC-Subtypen die $\gamma$-H2AXIntensität signifikant erhöht. Die
Autoren schlussfolgern, dass kurzzeitiges Fasten im Rahmen einer Chemotherapie hämatologische Nebenwirkungen abschwächt und zu einer schnelleren DNA-Reparatur und geringeren DNASchädigung in PBMC führt. Weitere Studien (u.a. mit längerem Fasten) sollten mehr Aufschlüsse über den möglichen Nutzen des Fastens in Verbindung mit einer Chemotherapie liefern.

de Groot S et al. The effects of short-term fasting on tolerance to (neo) adjuvant chemotherapy in HER2-negative breast cancer patients: a randomized pilot study. BMC Cancer. 2015;15:652

\section{Kommentar}

Die differenzielle Stressresistenz (DSR) beschreibt/den durch kurzzeitiges Fasten vermittelten Schutz des Organismus, nicht aber von Tumorzellen, vor zytotoxischen Einwirkungen. Langfristige Kalorienrestriktion verlängerte in verschiedenen Modellorgansimen das Leben und erhöhte die antioxidative Kapazität von Zellen, unter anderem durch Herabregulierung der RASund AKT-Signalwege. Ausgelöst durch die fastenbedingte Reduktion von Wachstumsfaktoren wie Glukose, Insulin und IGF-1 führt die verminderte Signaltransduktion zu einem Umschalten gesunder Zellen von Wachstum auf Zellerhalt und Stressresistenz. Tumorzellen aber, die durch genetische Mutationen auf Wachstum „programmiert" sind, profitieren von dieser Stressresistenz nicht, so die Hypothese.

DSR durch Fasten wurde inzwischen in experimentellen Studien mit verschiedenen Chemotherapeutika nachgewiesen. Tumorzellen scheinen durch Fasten auch in ihrem Wachstum gehemmt und gegenüber einer Chemotherapie sensibilisiert werden zu können. In der Studie von de Groot et al. wurde durch Fasten keine bessere Verträglichkeit der Chemotherapie erreicht. Als mögliche Erklärung wird das große Nebenwirkungsspektrum der Chemotherapie an- geführt, das die positiven Effekte des Fastens "verschleiert" haben könnte. Auch könnte das 24-stündige Fasten vor Chemotherapie für eine ausreichende Verminderung von Wachstumsfaktoren zu kurz gewesen sein. Bei den Patientinnen wurde zwar eine Senkung der IGF-1-Konzentrationen durch Fasten erreicht, eine Reduktion der Glukose- und Insulinspiegel durch Dexamethasongabe vor jeder Chemotherapie jedoch verhindert. Somit wurden wichtige metabolische Effekte aus den experimentellen Arbeiten nicht imitiert. Die Studie liefert aber Hinweise auf eine DSR der Blutzellen, insbesondere eine schnellere Erholung der Erythrozyten- und Thrombozytenzahlen. Interessant sind zudem die Ergebnisse zur $\mathrm{\gamma}$-H2AX-Anreicherung in Immunzellen, die auf eine beschleunigte Reparatur oder teilweise Verhinderung von DNA-Doppelstrangbrüchen in PBMC-Subpopulationen schließen lassen. Diese Ergebnisse wären konsistent mit einer verbesserten DNA-Reparatur durch eine Kalorienrestriktion.

In der Fastengruppe waren Insulin und IGF-1 gegenüber der Kontrollgruppe vermindert. Die Modulation der Insulin/IGF-1-Achse durch "fasting mimicking diets" ist ein interessanter Ansatz als Alternative zu Kalorienrestriktion/Fasten, vor allem bei drohender Mangelernährung. Zu solchen Diäten zählen protein- oder kohlenhydratbeschränkte ketogene Ernährungsformen, die metabolische Veränderungen ähnlich denen des Fastens induzieren sollen - idealerweise ohne die Gesamtenergieaufnahme einschränken zu müssen.

Weitere Untersuchungen müssten klare Kontraindikationen gegen Fasten definieren. Die Ergebnisse von de Groot et al. legen jedoch schon jetzt nahe, dass Fasten für bestimmte Patientengruppen eine potenziell effektive supportive Option darstellt persönliche Mitwirkung des Patienten vorausgesetzt.

\section{Literatur beim Verfasser}

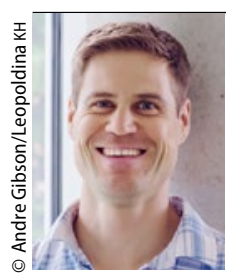

Dr. Rainer J. Klement Klinik für Strahlentherapie, Leopoldina Krankenhaus Schweinfurt 